\title{
Comparative Analysis of the Interaction Networks of HIV-1 and Human Proteins
}

\author{
Kyungsook Han* and Byungkyu Park \\ School of Computer Science and Engineering, Inha University, Inchon 402-751, Korea \\ khan@inha.ac.kr, bpark@inhaian.net
}

\begin{abstract}
Various interactions of human immunodeficiency virus type 1 (HIV-1) proteins with those of the host cell are known, but a large-scale network encompassing all known interactions of HIV-1 proteins and host cell proteins have not been analyzed or visualized. This is partly because large-scale interaction data are not readily available from public databases and individual literatures report small-scale interactions. NCBI recently released a database of all known interactions of HIV-1 proteins and human proteins. Now, a challenging task is to analyze all the interactions in a systematic way and to identify biologically meaningful interaction groups or patterns. This paper presents the development of a web-based system (http://hiv1.hpid.org) for visualizing and analyzing the large-scale interactions between HIV-1 and human proteins and the comparative analysis of the interactions. The whole interaction network contains 1,768 interactions of 65 different types with 810 human proteins. The analysis identified several interesting interaction groups from the comparative analysis of the interaction networks.
\end{abstract}

\section{Introduction}

Twenty years following its discovery, human immunodeficiency virus type 1 (HIV-1) remains a major threat to public health and a challenge for drug development [1]. Various interactions of HIV-1 proteins with those of the host cell are known, but a large-scale network encompassing all known interactions of HIV-1 proteins and host cell proteins have not been analyzed or visualized. This is partly because large-scale interaction data are not readily available from public databases and individual literatures report small-scale interactions.

NCBI recently released a database of all known interactions of HIV-1 proteins and human proteins (http://www.ncbi.nlm.nih.gov/RefSeq/HIVInteractions/). Now, a challenging task is to analyze all the interactions in a systematic way and to identify biologically meaningful interaction groups or patterns. We have developed a webbased system (http://hiv1.hpid.org) for systematically analyzing the large-scale interactions between HIV-1 and human proteins and the comparative analysis of the interactions. Comparative analysis of the interactions between HIV-1 and human proteins using the system revealed several interesting interaction groups. This is the

* Corresponding author.

Y. Shi et al. (Eds.): ICCS 2007, Part II, LNCS 4488, pp. 339-346, 2007.

(C) Springer-Verlag Berlin Heidelberg 2007 
first online system for interactively visualizing and analyzing the interactions of HIV-1 and human proteins. This paper presents the system and some analysis results.

\section{Methods}

We have constructed a web-based HIV-1 database (http://hiv1.hpid.org) for the analysis of the experimental data on the interactions between HIV-1 and human proteins. The experimental data were extracted from NCBI at http://www.ncbi.nlm.nih.gov/RefSeq/HIVInteractions. The whole interaction network contains 1,768 interactions between $11 \mathrm{HIV}-1$ proteins and 810 human proteins (Fig. 1). All the 65 types of protein-protein interactions shown in Table 1 were included in our analysis.

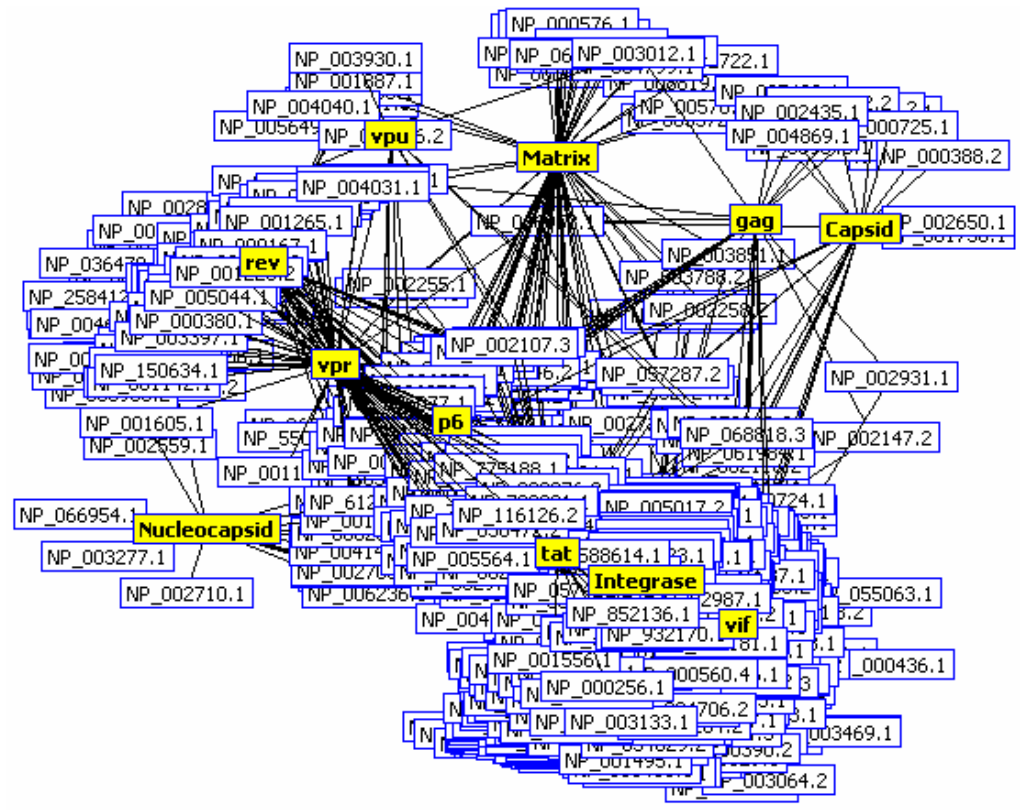

Fig. 1. The whole interaction network between 11 HIV-1 proteins and 810 human proteins. This network contains 1768 interactions of 65 different types. HIV-1 proteins are displayed in yellow in the networks.

Different HIV-1 proteins have different number of human proteins as their interacting partners (Fig. 2). HIV-1 tat protein has 667 human protein partners, which is the largest number of known human protein partners for a single HIV-1 protein. Table 2 shows the number of human proteins interacting with HIV-1 proteins, interactions that share human protein partners with other HIV-1 proteins, and the interactions of the "interact with" type that share human protein partners with other HIV-1 proteins. The numbers in the last row (total) represent the number of nonredundant occurrences (that is, duplicate occurrences of a same entity are counted 
only once). In the first and second columns, all types of interactions are considered, and the third column considers the interactions of the "interact with" type. The reason that the number of interactions differs from the number of proteins in the second column is because different types of interactions are counted more than once for a same pair of proteins. The interacting human proteins with an HIV-1 protein are visualized by our program called InterViewer [2, 3] as a star-shaped networks centered at the HIV-1 protein. We performed pairwise comparison of the interaction networks to find interaction groups.

Table 1. Types of interactions between HIV-1 and human proteins

\begin{tabular}{llll}
\hline interaction type & interaction type & interaction type & interaction type \\
\hline 1. acetylated by & 18. downregulates & 35. isomerized by & 52. releases \\
2. acetylates & 19. enhanced by & 36. modified by & 53. relocalizes \\
3. activated by & 20. enhances & 37. modifies & 54. repressed by \\
4. activates & 21. exported by & 38. modulated by & 55. requires \\
5. antagonized by & 22. fractionates with & 39. modulates & 56. sequesters \\
6. associates with & 23. imported by & 40. myristoylated by & 57. stabilizes \\
7. binds & 24. inactivates & 41. phosphorylated by & 58. stimulated by \\
8. co-localizes with & 25. incorporates & 42. phosphorylates & 59. stimulates \\
9. competes with & 26. increases & 43. polymerizes & 60. suppressed by \\
10. complexes with & 27. induces & 44. promotes binding to & 61. suppresses \\
11. cooperates with & 28. influenced by & 45. protects & 62. synergizes with \\
12. degraded by & 29. inhibited by & 46. recruited by & 63. transported by \\
13. degrades & 30. inhibits & 47. recruits & 64. ubiquitinated by \\
14. dephosphorylates & 31. inhibits induction of & 48. redistributes & 65. upregulates \\
15. depletes & 32. inhibits release of & 49. regulated by & \\
16. depolymerizes & 33. interacts with & 50. regulates & \\
17. deregulates & 34. interferes with & 51. regulates import of & \\
\hline
\end{tabular}

Table 2. The number of human proteins interacting with HIV-1 proteins, the interactions that share human protein partners with other HIV-1 proteins, and the interactions of the "interact with" type that share human protein partners with other HIV-1 proteins

\begin{tabular}{|c|c|c|c|c|c|}
\hline \multirow{2}{*}{\multicolumn{2}{|c|}{ HIV-1 proteins }} & \multirow{2}{*}{$\begin{array}{l}\text { Human } \\
\text { protein } \\
\text { partners }\end{array}$} & \multicolumn{2}{|c|}{$\begin{array}{l}\text { Interactions that share human } \\
\text { protein partners with other HIV-1 } \\
\text { proteins }\end{array}$} & \multirow{2}{*}{$\begin{array}{l}\text { Interactions of "interact } \\
\text { with" type that share } \\
\text { human protein partners } \\
\text { with other HIV-1 proteins }\end{array}$} \\
\hline & & & Interactions & Protein partners & \\
\hline \multirow{12}{*}{ gag } & capsid & 22 & 27 & 14 & 3 \\
\hline & nucleocapsid & 20 & 37 & 19 & 0 \\
\hline & matrix & 64 & 98 & 46 & 0 \\
\hline & p6 & 11 & 11 & 5 & 0 \\
\hline & gag Pr55 & 27 & 47 & 22 & 1 \\
\hline & pol & 69 & 114 & 61 & 2 \\
\hline & rev & 55 & 56 & 33 & 1 \\
\hline & tat & 667 & 291 & 178 & 47 \\
\hline & vif & 56 & 98 & 47 & 39 \\
\hline & vpr & 128 & 157 & 93 & 4 \\
\hline & vpu & 18 & 24 & 13 & 1 \\
\hline & total & 810 & 960 & 204 & 98 \\
\hline
\end{tabular}




\section{Results and Discussion}

From the comparative analysis of HIV-1 interaction networks, we identified several interaction groups of human proteins. Human proteins in a same interaction group always have the same set of HIV-1 proteins as their interacting partners. There are a total 23 interaction groups, 2 in network A, 1 in network B, 7 in network C, and 13 in network D (Fig. 3). Each interaction group of human proteins is shown in a red round box, and interacting HIV-1 proteins are shown in yellow round boxes.

The network in Fig. 3A shows 2 groups of human proteins with 5 interacting HIV-1 proteins. 2 human proteins in a group, NP_002736 and NP_002737, have the same interacting partners of HIV-1 proteins p6, tat, rev, vif, and matrix. Human proteins NP_002736 and NP_002737 are mitogen-activated protein kinase (MAPK) 1 and 3, respectively. MAPK has been shown to regulate HIV-1 infectivity by phosphorylating vif [4]. Phosphorylation of vif by a serine/threonine protein kinase(s) plays an important role in regulating HIV-1 replication and infectivity. The gagderived protein $\mathrm{p} 6$ of HIV-1 plays a crucial role in the release of virions from the membranes of infected cells [5]. Three human proteins in the group \{NP_002256, NP_002261, NP_002262\} in Fig. 3A have the same interacting partners of tat, rev, integrase, matrix, and vpr. NP_002256, NP_002261, and NP_002262 are karyopherin beta 1 , transportin 1, RAN binding protein 5 , respectively.

Fig. 3B shows an interaction group of 8 human proteins $\{$ NP_002145, NP_002146, NP_004125, NP_005336, NP_005337, NP_005338, NP_006588, NP_068814\}, all interact with vpr, matrix, tat, and gag. The 8 human proteins are the members of the Hsp70 protein family, and by controlled binding and release, facilitate the folding, oligomeric assembly-disassembly, and intracellular transport of protein complexes.

Fig. 3C shows 7 interaction groups of human proteins, each with a same set of 3 HIV-1 protein partners. Among the interaction groups, the group of 40 human proteins interacting with integrase, vif and tat is the largest. The network in Fig. 3D shows 13 interaction groups of human proteins. The human proteins in a same interaction group share a same set of 2 interacting partners. The group interacting with tat and vpr is the largest, containing 42 human proteins.

Fig. 4 shows an interaction network of HIV-1 and human proteins, consisting of interactions of "interact with" type only. The network shows 98 interactions between 11 HIV-1 proteins and 49 human proteins. The human proteins shared by the vif and tat genes (enclosed in a red box in the network) are proteasome (prosome, macropain) subunits of a highly ordered ring-shaped 20S core structure.

Fig. 5 shows the number of interacting human proteins of each HIV-1 protein and the interactions of the "interact with" type that share human protein partners with other HIV-1 proteins. HIV-1 tat protein has the largest number of interacting human proteins as well as the largest number of interactions of the "interact with" type.

In summary, 6 out of total 810 human proteins $(0.7 \%)$ interact with exactly 5 HIV-1 proteins (the interaction groups in Fig. 3A), 12 human proteins (1.5\%) interact with exactly 4 HIV-1 proteins (Fig. 3B), 81 human proteins (10\%) interact with exactly $3 \mathrm{HIV}-1$ proteins (the interaction groups in Fig. 3C), 105 human proteins (13\%) interact with exactly 2 HIV-1 proteins (Fig. 3D), and 606 human proteins $(74.8 \%)$ interact with only one HIV-1 protein. There is no human protein that interacts with more than 5 HIV-1 proteins. 


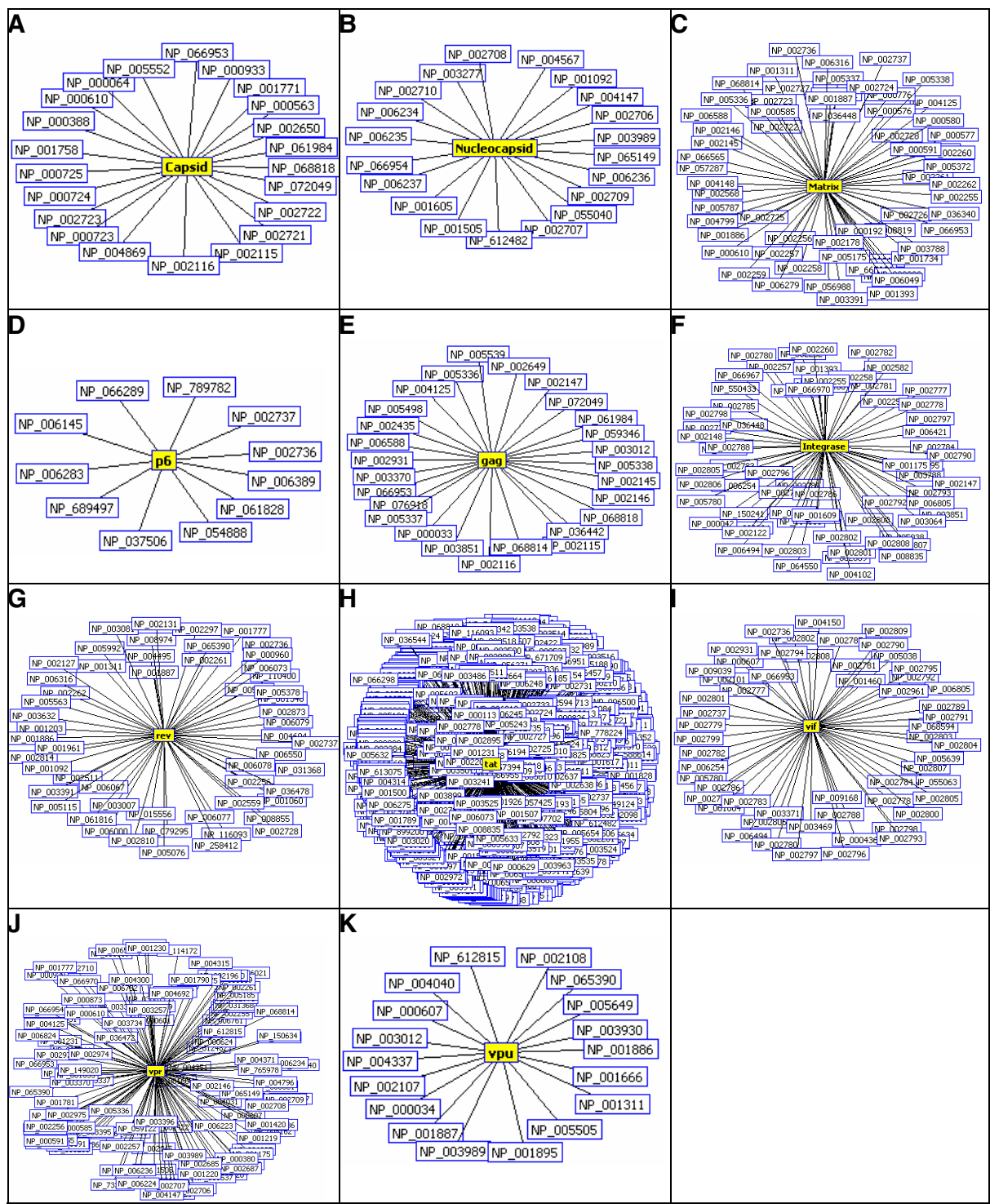

Fig. 2. The interaction networks between HIV-1 and human proteins, in which HIV-1 proteins and human proteins are displayed in yellow and white nodes, respectively. (A) HIV-1 capsid protein interacting with 22 human proteins. (B) HIV-1 nucleocapsid protein interacting with 20 human proteins. (C) HIV-1 matrix protein interacting with 64 human proteins. (D) HIV-1 p6 protein interacting with 11 human proteins. (E) HIV-1 gag Pr55 protein interacting with 27 human proteins. (F) HIV-1 integrase protein interacting with 69 human proteins. (G) HIV-1 rev protein interacting with 55 human proteins. (H) HIV-1 tat protein interacting with 667 human proteins. (I) HIV-1 vif protein interacting with 56 human proteins. (J) HIV-1 vpr protein interacting with 128 human proteins. (K) HIV-1 vpu protein interacting with 18 human proteins. All the networks are star-shaped networks centered at an HIV-1 protein. 


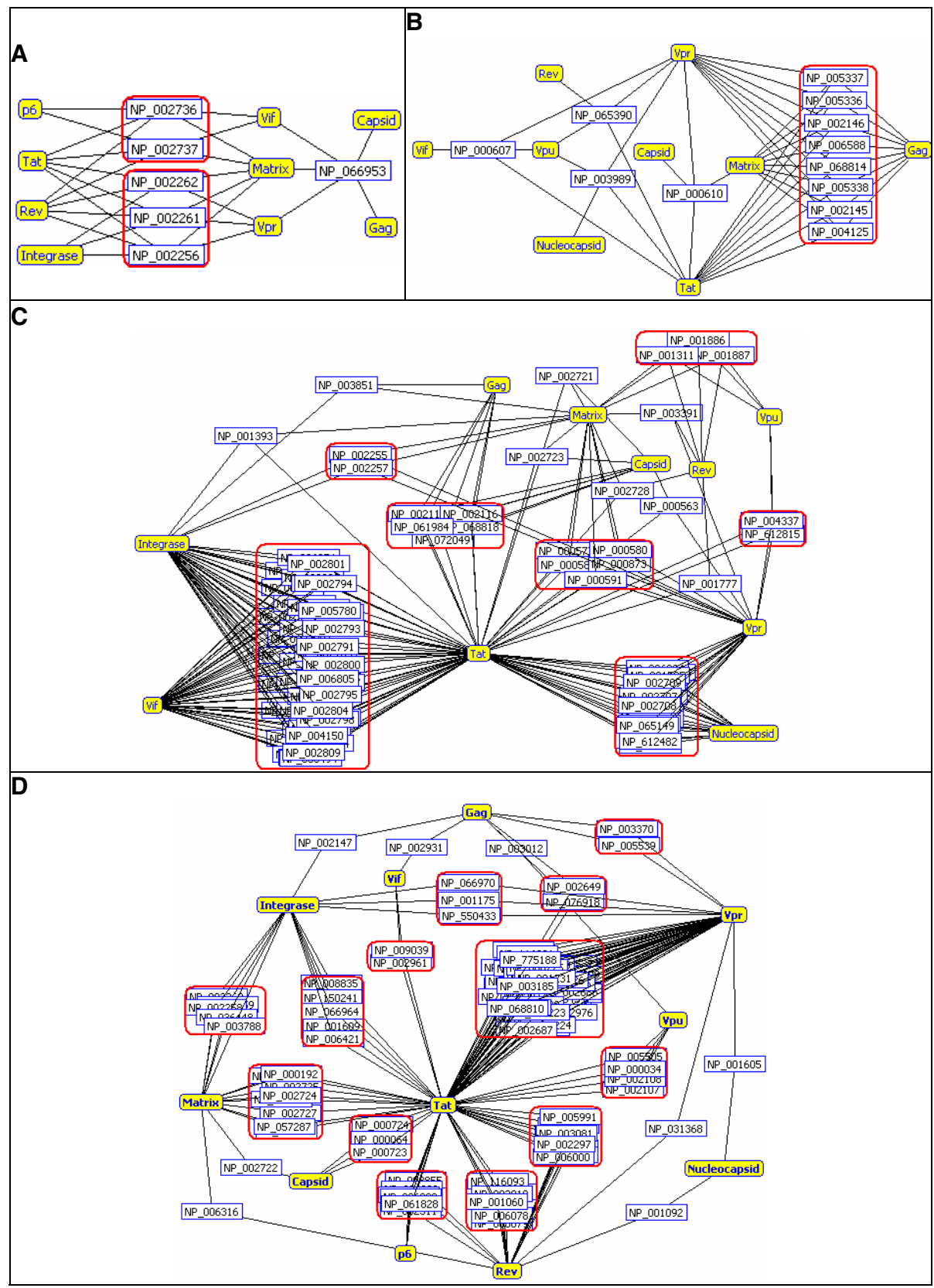

Fig. 3. The interaction groups of human proteins identified from the analysis of the interactions between HIV-1 and human proteins. Proteins in a same interaction group always have the same set of interacting partners. There are a total 23 interaction groups, 2 in network A, 1 in network $\mathrm{B}, 7$ in network $\mathrm{C}$, and 13 in network $\mathrm{D}$. Each interaction group of human proteins is shown in a red round box, and interacting HIV-1 proteins are shown in yellow round boxes. 


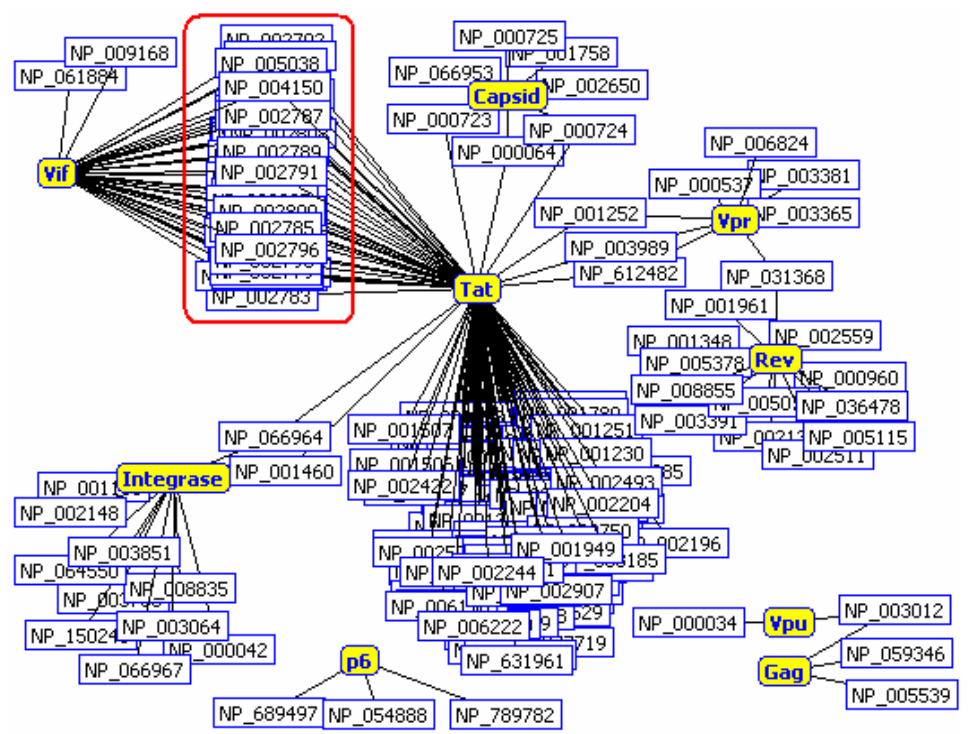

Fig. 4. Interaction network of HIV-1 and human proteins, consisting of interactions of "interact with" type only. The network shows 98 interactions between $11 \mathrm{HIV}-1$ proteins and 49 human proteins. The human proteins shared by the vif and tat genes (enclosed in a red box in the network) are proteasome (prosome, macropain) subunits of a highly ordered ring-shaped $20 \mathrm{~S}$ core structure.

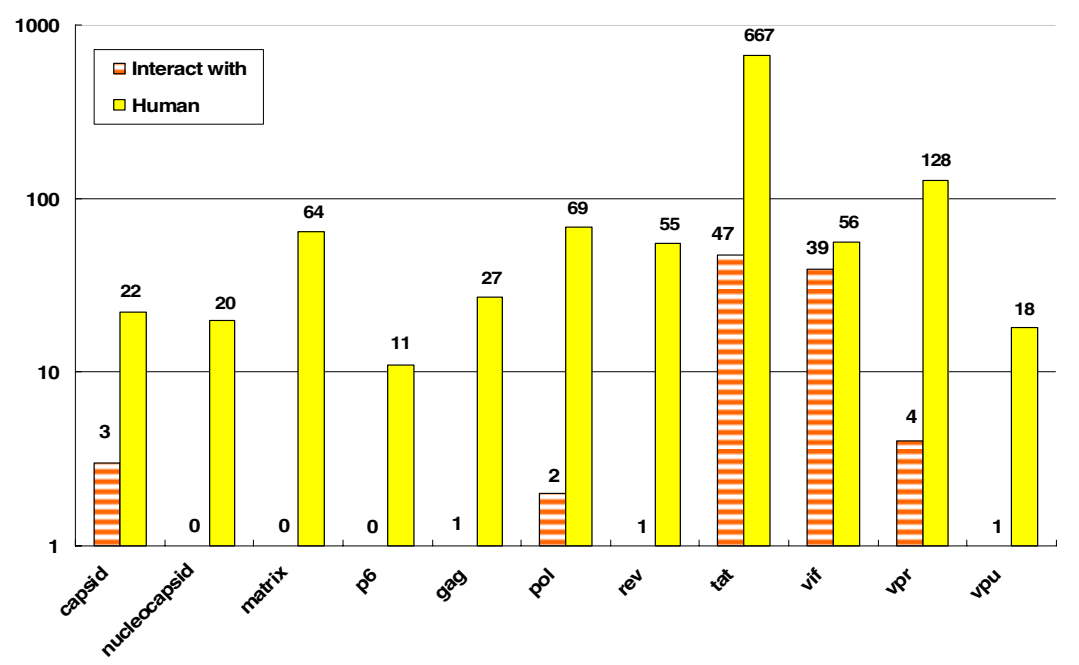

Fig. 5. The number of interacting human proteins of each HIV-1 protein (yellow bars) and the interactions of the "interact with" type that share human protein partners with other HIV-1 proteins (orange hatched bars) 


\section{Conclusion}

Most of the interactions between virus and host cells are complex and have not been fully understood despite the substantial discoveries in recent years. Investigating virus-host interactions is important for understanding viral replication and for identifying new targets with potential for drug intervention. We have constructed a web-based HIV-1 database (http://hiv1.hpid.org) for the comparative analysis of the experimental data on the interactions between HIV-1 and human proteins. Comparative analysis of the interactions between HIV-1 and human proteins using the system revealed several interesting interaction groups. Our work can be extended in several directions. First, biological experiments can be performed to identify functions or other biological properties common to all proteins in the same interaction group. Second, our study analyzed the interactions between HIV-1 and human proteins, but can be expanded to include the interactions between human proteins. Finally, our system should be updated to include the interaction data for the HIV-1 nef protein, which has been added to the HIV-1 database of NCBI very recently. We believe that this is the first online system for the comparative analysis of the interactions networks of HIV-1 and human proteins and that it is a valuable tool for scientists in the field of protein-protein interactions and HIV/AIDS research.

\section{Acknowledgements}

This work was supported by the Korea Research Foundation Grant funded by the Korean Government (KRF-2006-D00038) and in part by MOST (KOSEF) through the Systems Bio-Dynamics Research Center.

\section{References}

1. Trkola, A.: HIV-host interactions: vital to the virus and key to its inhibition. Current Opinion in Microbiology 7 (2004) 555-559

2. Han, K., Ju, B., Jung, H.: WebInterViewer: Integrated Framework for Visualizing and Analyzing Molecular Interaction Networks. Nucl. Acids Res. 32 (2004) W89-W95

3. Ju, B.-H., Han, K.: Complexity Management in Visualizing Protein Interaction Networks. Bioinformatics 19 (2003) i177-i179

4. Yang, X., Gabuzda, D.: Regulation of human immunodeficiency virus type 1 infectivity by the ERK mitogen-activated protein kinase signaling pathway. Journal of Virology 73 (1999) 3460-3466

5. Müller, B., Patschinsky, T., Kräusslich, H.G.: The Late-Domain-Containing Protein p6 Is the Predominant Phosphoprotein of Human Immunodeficiency Virus Type 1 Particles. Journal of Virology 76 (2002) 1015-1024 